\title{
Coupling between propagated modes in optical fibres with periodical structures
}

\author{
Y.W. Bobitski, V.M. Fitio, S.Yu. Lebid \\ National University "L'vivska Polytechnica", 12 S.Bandery Str., 79013 L'viv, Ukraine, \\ Tel: 80322 727747; E-mail: bobitski@polynet.lviv.ua, Polyana@polynet.lviv.ua
}

\begin{abstract}
Theoretical analysis was conducted with the purpose for further development of spectral-sensitive elements for fibre optic devices including sensors of physical magnitudes. Forecasting performances of such elements is conducted. Analysis has shown that coupling coefficient between propagating modes (incident and reflected) of fibre cladding grating is sufficient for sensitive elements manufacturing because on fibre cladding it is possible to achieve significant equivalent periodic modification of refraction index not taking into consideration that wave amplitude in the cladding is significantly less than in the core. Such gratings can be created by holographic method using photopolymeric media and lasers working in blue-green area of spectrum. The case of the corrugated or sinusoidal boundary between fibre core and cladding is also possible, and between cladding and external medium under investigation, for example with water, where various substances are dissolved, influencing on refraction and absorption of light waves propagating in the fibre. The results of theoretical researches can be used for developing devices with periodic structures on optical fibres.
\end{abstract}

Keywords: grating, optical fibre, refraction index, coupling coefficient.

Paper received 26.03.01; revised manuscript received 09.04.01; accepted for publication 13.07.01.

\section{Introduction}

Periodic structure in optical fibre (fibre core or cladding grating) leads to coupling between propagated modes. The same properties may be inherent to a corrugated boundary with sinusoidal profile between core and cladding or between cladding and investigated substance. Therefore, for creation and forecasting operation performances of fibre optic devices based on periodic structures, it is important to fulfil the analysis of distribution of light in such fibres and to evaluate their efficiency (to calculate coupling coefficient between modes). It is easy to calculate coupling coefficients between distributed modes for single-mode fibres, but this technique can be distributed for multi-mode fibres. For this purpose, it is necessary to know functions, that describe extension modes [1].

At present there is a significant number of works devoted to periodic structures on an optical filament [1,511]. Such periodic structures are formed on a core of a filament, for example, using an eximer $\mathrm{KrF}$ laser, and modification of refraction index at the best reaches $10^{-6} \ldots 10^{-3}[9]$. Formation technology of periodical struc- tures on the fibre core is enough complicated and requires both unique lasers with generation in UV range and special sensitive fibres. Therefore, there appears the next question: is it possible to achieve necessary coupling between modes to receive desirable reflection coefficient for other types of periodical structures (for fibre cladding gratings, for corrugated boundary between core and cladding of optical fibre or between cladding and external medium)?

\section{Analysis of light propagation in single-mode optical fibers}

Let us make a calculation of basic modes characteristics for weakly guiding single-mode fibres taking into consideration the theory of their propagation in such fibre [4]. The constant of propagation $\beta$ of the main mode exists in the interval between two extremums determined by meanings of $\beta$ for plane waves propagating in $Z$ direction in boundless homogeneous media with refraction indexes equal to the maximal and minimal meanings of the fibre refraction index $n(r)$. If such meanings will be presented as $n_{\max }$ - maximal meaning of the refraction 


\section{Y.W. Bobitski et al.: Coupling between propagated modes in optical fibres ...}

index $n(r), n_{\min }-$ minimal meaning of the refraction index $n(r)$, the $\beta$ will be in the range:

$\frac{2 \pi n_{\max }}{\lambda}<\beta<\frac{2 \pi n_{\min }}{\lambda}$,

where $\lambda$ is a wavelength in vacuum. Taking into consideration that fibres are weakly guided, that is $n_{\max } \cong n_{\min }$, then from ( 1 ) follows: $\beta \cong \frac{2 \pi n}{\lambda}$, which corresponds to a constant of propagation of plane wave in $Z$-direction in endless medium with refractive index $n_{\max } \geq n \geq n_{\min }$.

Polarization properties of fibre structure weakly influence on the field in the fibre because $n_{\text {max }} \cong n_{\min }[11]$. Correspondingly, an optic wave propagated in the fibre could not be sensitive to the polarization effects. Therefore, wave propagation in weakly guided fibres can be described by scalar wave equation:

$$
\left\{\frac{\partial^{2}}{\partial r^{2}}+\frac{1}{r} \frac{\partial}{\partial r}+k^{2}(r)\right\} \psi(r)=\beta^{2} \psi(r),
$$

where is a $\psi(r)$ scalar function corresponding to electric field strength in the fibre and $k(r)$ can be determined by expression:

$$
k(r)=\frac{2 \pi n(r)}{\lambda}
$$

Them main mode described by solution of equation (2) corresponding to maximal $\beta$ at that $\psi(r)$ does not depend on the polar angle $\phi$. Satisfactory approximation can be obtained for the field $\psi(r)$ described by the function:

$$
\psi(r) \cong C \exp \left(-\frac{1}{2}\left(\frac{r}{r_{0}}\right)^{2}\right)
$$

where $r_{0}$ is a spot size determined by variation method and the constant $C$ determined from the condition of normalization:

$$
2 \pi \int_{0}^{\infty} r \psi^{2}(r) d r=1 .
$$

The function (4) is a solution of the equation (2), and it can be used as a trial function for a stationary expression of the constant of propagation $\beta$, when $r_{0}$ being chosen from the condition of providing maximal $\beta$ [12] corresponding to the main mode. As a result of simple substitution there appears expression for $\beta$ :

$$
\beta^{2}=\frac{\int_{0}^{\infty}\left\{-\left(\frac{d \psi}{d r}\right)^{2}+k^{2}(r) \psi^{2}\right\} r d r}{\int_{0}^{\infty} r \psi^{2} d r} .
$$

In concordance with correlation (6) dependence $\beta^{2}=f\left(r_{0}\right)$ is created, it is obvious that $r_{0}$ changes in the range of $\rho-\delta \rho \leq \rho \leq \rho+\delta \rho$, at the same time $\delta \rho$ is chosen in such range that on the curve $\beta^{2}=f\left(r_{0}\right)$ we can see maximum (see Fig.1). For a step-index fibre [4], $r_{0}$ corresponds to the core radius, what we can prove by substitution of (4) into (6) and equaling the first derivative of the expression (6) on $r_{0}$ to zero:

$\frac{\partial \beta^{2}}{\partial r_{0}}=0$

Knowing $r_{0}$ and $\beta$, and taking into consideration (5), we can completely characterize the field of an optical wave propagating in single mode fibre.

Periodical structures on the optical fibre can be of different types. Calculation of coupling coefficient between coupled modes for the case when periodic structure is situated on the fibre core is necessary for us to compare it with coupling coefficient for cases when different periodic structures are situated on the fibre cladding. It is obvious that cases of corrugated boundaries core-cladding and cladding-cladding, when claddings consist of different materials, are practically equivalent, and we provide calculation only for the first case. So, we calculate coupling coefficients between modes (incident and reflected waves) for a single mode fibre using the correlation obtained in [5].

$\chi=\frac{\pi}{\beta} \int_{0}^{\infty} r \psi^{2}(r) k_{0}^{2}(r) m(r) d r$

where $\chi$ is a coupling coefficient between an incident wave on periodical structure and reflected wave from this structure for the single mode fibre, and $m(r)$ is a coefficient of modulation refraction indexes describing strength of periodical structure

$$
n(r, z)=n_{0}(r)\left[1+m(r) \cos \left(\frac{2 \pi}{\Lambda} z\right)\right]
$$

Knowing $\psi(r), r_{0}, \beta, m(r), n(r)$ we can calculate the coupling coefficient $\chi$ between modes for different periodical structures.

\section{Calculation of optical wave propagation in single-mode fibres with gratings on the core and cladding}

At the beginning, we calculate parameters $r_{0}$ and $\beta$ for single mode fibre, considering that refraction indexes of fibre core and cladding are constant and equal to $n_{1}$ and $n_{2}$, respectively. Substituting (4) into (6) we receive the following expression: 


$$
\beta^{2}=\frac{-0.5+\left(\frac{2 \pi n_{1}}{\lambda}\right)^{2} \int_{0}^{\rho} \exp \left[-\left(\frac{r}{r_{0}}\right)^{2}\right] r d r+\left(\frac{2 \pi n_{2}}{\lambda}\right)^{2} \int_{\rho}^{\infty} \exp \left[-\left(\frac{r}{r_{0}}\right)^{2}\right] r d r}{\int_{0}^{\infty} \exp \left[-\left(\frac{r}{r_{0}}\right)^{2}\right] r d r} .
$$

For the step index fibre $r_{0}=\rho$, what is confirmed also by analogous calculation in accordance to the formula (6a). Using the correlation (8) we can calculate the coupling coefficient $\chi$ for a fibre core grating. In such case the formula (8) will have a form:

$\chi=\frac{\pi}{\beta} \int_{0}^{\rho} r \psi^{2}(r) k_{0}{ }^{2}(r) m d r$.

From the correlation (8a) cropped up that coupling coefficient is proportional to $m$. Therefore it is sufficient to calculate $\chi$ only for, e.g., $m=0.001$. Substituting data derived from correlations $(5,6)$ into the formula $(8 a)$, we get $\chi=1851 \mathrm{~m}^{-1}$.

According the formulation (8) we can also calculate the coupling coefficient $\chi$ for periodic grating on the fibre cladding. The formulation (8) modifies and appears:

$\chi=\frac{\pi}{\beta} \int_{\rho}^{\infty} r \psi^{2}(r) k_{0}{ }^{2}(r) m d r$.

Calculation of the coupling coefficient using the latter formula was fulfilled also for $m=0.001$, which for our case was equal to $1073 \mathrm{~m}^{-1}$. So, comparing coupling coefficients on the fibre core and cladding at similar $m$, we can conclude that they are balanced. But taking into account that the fibre cladding can be made of photosensitive material, for e.g. photopolymer, one can record a grating on it by holographic methods using lasers in blue-

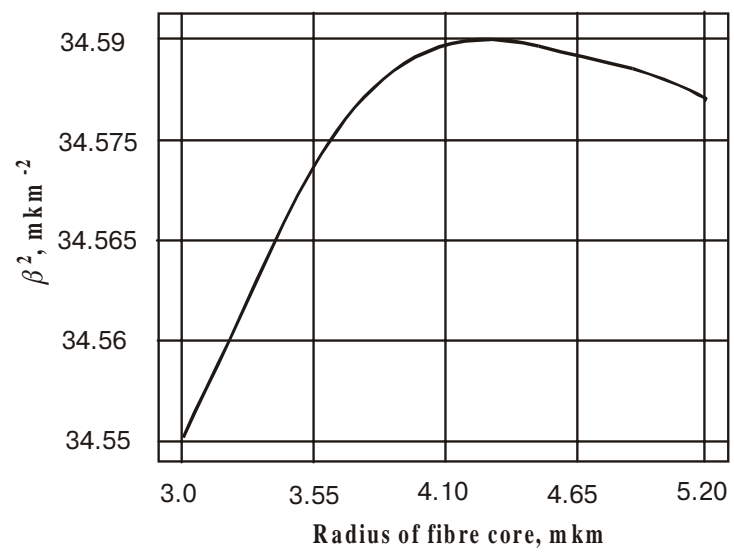

Fig. 1. Dependence of the square of propagation constant $\beta^{2}$ on radius $r$ of the fibre with refraction green spectral range, where $m$ can have meanings 0.01 [13]. Thus, such grating on the fibre cladding can be more effective for its operation than gratings on the fibre core.

\section{Calculation of a coupling coefficient for an optic fibre with corrugated boundaries}

Optical fibres with corrugated claddings can be effectively used in different sensors because of these can have contact with investigated media, optical characteristics of which (refraction and absorption indexes) directly influence on reflection coefficient of the grating. Optical fibre with corrugated boundary between core and cladding is schematically shown in Fig.2. Such boundary can be mathematically expressed by the following way:

$r(z)=\rho\left[1+m \cos \left(\frac{2 \pi}{\Lambda} z\right)\right]$.

Then refraction index as a function of $r$ in such optic fibre will appear in the following way:

$$
n(r)= \begin{cases}n_{2}, & \mathrm{r}>\rho\left[1+m \cos \left(\frac{2 \pi}{\Lambda} z\right)\right], \\ n_{1}, & \mathrm{r}<\rho\left[1+m \cos \left(\frac{2 \pi}{\Lambda} z\right)\right] .\end{cases}
$$

On the basis of the formula (9) we can deduce the following correlation:

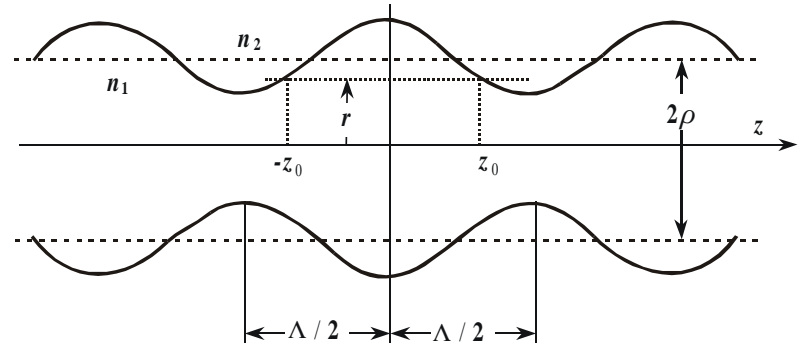

Fig. 2. Sinusoidal boundary between core and cladding of optical fibre. 
$\cos \left(\frac{2 \pi}{\Lambda} z\right)=\frac{r-\rho}{m \rho}$.

The latter equation can be solved concerning $z_{1,2}$, when radius of the boundary between cladding and core equals $r$.

$$
z_{1,2}= \pm \frac{\Lambda}{2 \pi} \arccos \left(\frac{r-\rho}{m \rho}\right)= \pm z_{0} .
$$

Using the letter correlation one can find coefficients of complex Fourier series that express periodic changes of the fibre refraction index along the dimension $Z$ in accordance with (9) and (10) formulae.

$$
\begin{aligned}
& C_{k}(r)=\frac{1}{\Lambda} \int_{-\Lambda / 2}^{\Lambda / 2} n(r) \exp \left(-j \frac{2 \pi}{\Lambda} k z\right) d z= \\
& =\frac{1}{\Lambda} \int_{-\Lambda / 2}^{-z_{0}} n_{2} \exp \left(-j \frac{2 \pi}{\Lambda} k z\right)+ \\
& +\frac{1}{\Lambda} \int_{-z_{0}}^{z_{0}} n_{l} \exp \left(-j \frac{2 \pi}{\Lambda} k z\right)+\frac{1}{\Lambda} \int_{z_{0}}^{\Lambda / 2} n_{2} \exp \left(-j \frac{2 \pi}{\Lambda} k z\right)
\end{aligned}
$$

From the latter formula (13), in the result of integration, we can directly find the coefficient $C_{k}(r)$.

$$
C_{k}(r)=\frac{n_{1}-n_{2}}{\pi k} \sin \left[k \arccos \left(\frac{r-\rho}{m \rho}\right)\right] .
$$

We are interested mainly in $C_{1}(r)$, that in accordance to the formula (14) can be expressed in the following way:

$$
C_{1}(r)=\left\{\begin{array}{l}
\frac{n_{1}-n_{2}}{\pi} \sqrt{1-\left(\frac{r-\rho}{m \rho}\right)^{2}}, \quad \rho-m \rho \leq r \leq \rho+m \rho ; \\
0, \quad r<\rho-m \rho, \quad r>\rho+m \rho .
\end{array}\right.
$$

The coefficient $C_{0}(r)$ is found by substitution of the formula (13) where $k=0$, so

$$
C_{0}(r)=\left\{\begin{array}{l}
\frac{n_{1}-n_{2}}{\pi} \arccos \left(\frac{r-\rho}{m \rho}\right)+n_{2}, \quad \rho-m \rho \leq r \leq \rho+m \rho ; \\
n_{1}, \quad r<\rho-m \rho ; \\
n_{2}, \quad r>\rho+m \rho .
\end{array}\right.
$$

Thus, in a general case, dependence of refraction index on $r$ will have the following appearance:

$$
\begin{aligned}
& n(r)=C_{0}(r)+2 C_{1}(r) \cos \left(\frac{2 \pi}{\Lambda} z\right)+\ldots \\
& \ldots+2 C_{k}(r) \cos \left(\frac{2 \pi}{\Lambda} k z\right)+\ldots= \\
& =C_{0}(r)\left[1+2 \frac{C_{1}(r)}{C_{0}(r)} \cos \left(\frac{2 \pi}{\Lambda} z\right)+\ldots\right]= \\
& =C_{0}(r)\left[1+m(r) \cos \left(\frac{2 \pi}{\Lambda} z\right)+\ldots\right]
\end{aligned}
$$

Hence, $m(r)=2 \frac{C_{1}(r)}{C_{0}(r)}$. Substituting this meaning $m(r)$ into the expression (8) that have the following form in this case:

$\chi=\frac{(2 \pi)^{3}}{\beta \lambda^{2}} \int_{\rho-m \rho}^{\rho+m \rho} r \psi^{2}(r) C_{0}(r) C_{1}(r) d r$

In accord with the formula (18) we can calculate coupling coefficient between coupled modes for corrugated boundaries between the fibre core and cladding. The plot of dependences of the coupling coefficient between modes when changing $m$ in the range from 0.001 to 0.1 , for $n_{1}=1.453, n_{2}=1.45$ is shown in Fig. 3 .

The coupling coefficient between modes $\chi$ for $m=$ 0.001 equals $4.436 \mathrm{~m}^{-1}$. From such a plot it follows that to achieve desirable coupling coefficient value $\chi=100 \ldots$ $400 \mathrm{~m}^{-1}$ are necessary [6] to have a modulation coefficient value $m=0.02 \ldots 0.08$.

For calculation of coupling coefficient between modes at corrugated boundary between fibre cladding and external investigated medium we can use the expression (18). It is necessary to take into consideration that in our case the middle radius of corrugated boundary will differ from $\rho$, and such value will be marked as $r_{1}$. For different refraction indexes of external medium $n_{s}$, it is necessary to calculate $r_{0}$ that already will not be equal to the core radius and also calculate the propagation constant $\beta$. Such values $r_{0}$ and $\beta$ can be found by the method described in [4]. So

$\chi=\frac{(2 \pi)^{3}}{\beta \lambda^{2}} \int_{\eta-m \eta}^{\eta+m \eta} r \psi^{2}(r) C_{0}(r) C_{1}(r) d r$

where $C_{0}(r), C_{1}(r)$ are calculated using expressions (15) and (16) at displacement $\rho$ on $r_{1}, n_{1}$ onto $n_{2}$ and $n_{2}$ onto $n_{s}$. Calculation provided for $r_{1}=6 \mu \mathrm{m}, n_{s}=1.33$ and $n_{s}=135$. At this will received meaning $r_{0}=3.15 \mu \mathrm{m}$, and $\beta=5.879$. Dependence of coupling coefficient between modes on the value of modulation $m$ of corrugated surface for two meanings of refraction indexes is shown in Fig. 3. It follows from this figure that for corrugated cladding one can also reach greater coupling coefficient at modulation of external diameter of cladding in the range of $0.02 \ldots 0.08$. Moreover, the coupling coefficient 
Y.W. Bobitski et al.: Coupling between propagated modes in optical fibres ...

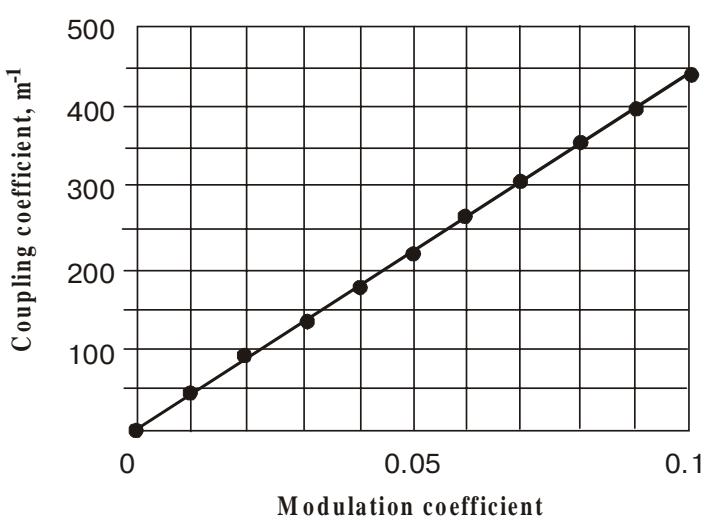

b)

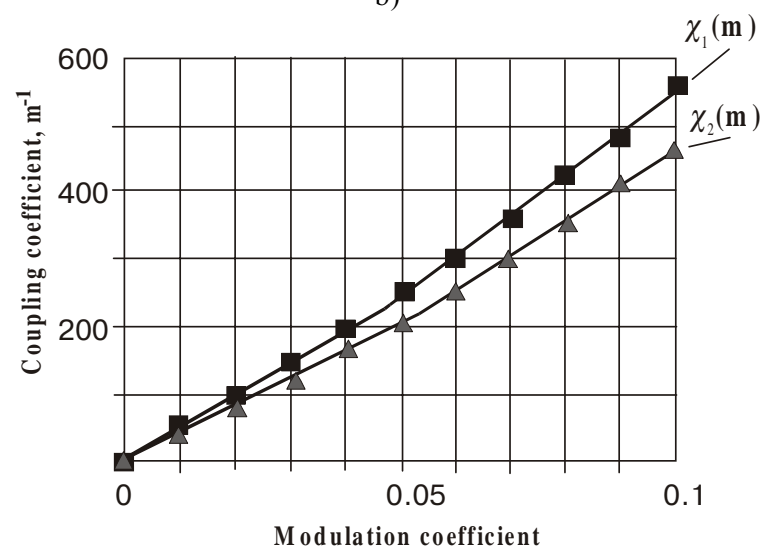

Fig. 3. Plots of dependences of coupling coefficients between modes on modulation coefficient $m(r)$ : a) coupling coefficient for corrugated grating core-cladding; b) $\chi_{1}(m)$ and $\chi_{2}(m)$ - coupling coefficients for corrugated gratings on the cladding of optic fibre at $n_{S}=1.33, n_{S}=1.35$.

between modes of such fibre is very sensitive to the change of index $n_{S}$ of external medium.

\section{Discussion of results}

Analysis has shown that the coupling coefficient for waves on the cladding and core of the fibre are balanced. But, it should be noticed that on the fibre cladding some grating could be formed by holographic methods using available lasers and photosensitive polymers. Such technology is very simple and can be realized in production of different optoelectronic devices. If external boundary of fibre cladding is corrugated, then such element with a periodic structure can be used as sensor for measurements of different characteristics of water and water solutions, which are bound with refraction and absorption indexes. Calculations showed that coupling coefficient between modes for corrugated claddings is sufficiently sensitive to changes of refraction index of medium in which fibre exists. But, production of such optoelectronic elements with periodic structures requires a lot of technological investigations.

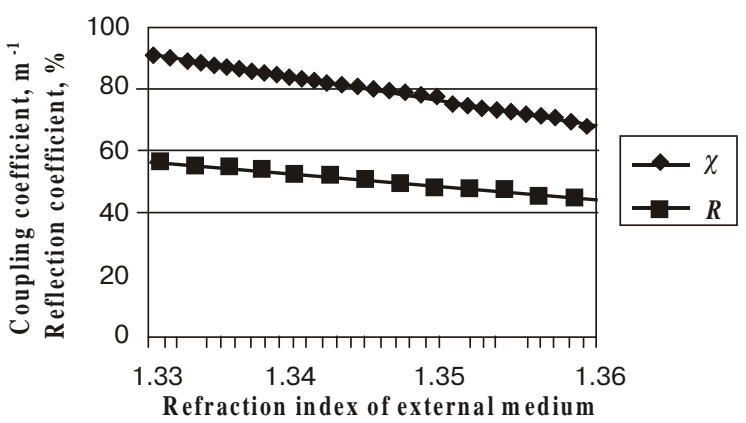

Fig. 4. Plots of dependences of coupling $(\chi)$ and reflection $(R)$ coefficients on a refraction index of medium under investigation for corrugated boundary cladding-external medium.

\section{References}

1. A. Yariv, P. Yeh, Optical waves in crystals, A WileyInterscience Publication. John Wiley \& Sons, New York, (1984).

2. B.J.-C. Deboux, E. Lewis, P.J. Scully, R. Edwards, A novel technique for optical fiber $\mathrm{pH}$ sensing based on methylene blue adsorption // IEEE J. of Lightwave Tech., 13(7), pp. 1407-1414 (1995).

3. R. Edwards, E. El-Saadawy, M. Gander, P.J. Scully, A. Young, P. Baker. J. Grabowski, The development of a biological toxicity based test for water quality using a fibre optic sensor // Inter. Symp. On Analytical Chem., Hull (UK), July, 1995.

4. A.W. Snyder, Understanding monomode optical fibers // Proceedings IEEE, 69(1), pp. 6-13 (1981).

5. Y. Bobitski, V. Fitio, S. Lebid, T. Wanchytski, Reflection spectrum calculation in optical fiber periodical structures (PS) // Proc. 3rd International Conf. MECHATRONIKA'97, Warszawa (Poland), pp. 775-781 (1997).

6. S. Lebid, Y. Bobitski, V. Fitio, T. Fityo, Spectrum of Bragg grating reflection coefficient (RC) in optical fiber // Proc. of SPIE, 3291, pp. 165-173 (1998).

7. S. Juma, Bragg gratings boost data transmission rates // Laser Focus World. Fiberoptic Components. Handbook., November, pp. 1-5 (1996).

8. IBM Holographic Optical Storage Team, Holographic storage promises high data density // Laser Focus World, November, pp. 81-86 (1996).

9. V. Mizrahi, P. J.Lemaire, T. Erdogan, W.A.Reed, D. J DiGiovanni, R. M.Atkins, Ultraviolet laser fabrication of ultrastrong optical fiber gratings and of germanium doped channel waveguides // Appl.Phys.Lett., 63(13), pp. 17271729 (1993)

10. E. D. Jungbluth, Phase-mask photolithography makes Bragg gratings in fiber // Laser Focus World, May, pp. 33-35 (1993).

11. A.W. Snyder, J.D. Love, Optical Waveguide Theory, Chapman and Hall, London, (1981).

12. A.W. Snyder, R.A. Sammut, Fundamental (HE) modes of graded optical fibers // J. Opt. Soc. Amer., 69, pp. 1663-1671 (1979).

13. T. N. Smirnova, E. S. Gulnasarov, E. A. Tikhonov, Optical nonlinearity and holographic recording of stable periodic structures in polimeric photorefractive media // Proc. of SPIE, 1017, pp. 190-192 (1988). 